\title{
Alterazioni eritrocitarie in emodialisi
}

\author{
P. Calzavara
}

\section{U.O. Nefrologia e Dialisi - Treviso}

li eritrociti (E) circolanti, nel soggetto normale, hanno un diametro medio di $8 \mu \mathrm{m}$, spessori medi di $1 \mu \mathrm{m}$ al centro e di 2.5 ai bordi e volume medio di $90 \mu \mathrm{m} 3$.

Al microscopio ottico, se colorati con il May Grünwald-Giemsa, si tingono di rosa-arancione e appaiono come elementi di forma circolare con un area centrale più pallida; al microscopio elettronico a scansione rivelano con maggior chiarezza il tipico aspetto biconcavo o le eventuali alterazioni morfologiche (Fig. 1).

Queste cellule, la cui vita media è di 120 giorni in condizioni normali, derivano dal proeritroblasto attraverso una serie di tappe di amplificazione e di maturazione. Una volta completato il loro ciclo vitale vengono catturati e fagocitati dalle cellule reticolo-endoteliali soprattutto del midollo osseo e della milza (1).

La membrana eritrocitaria è costituita da un doppio strato lipidico con interposizione di componenti proteiche e glucidiche. Alterazioni strutturali della membrana, da stress meccanici, chimico-fisici, immunologici sono in grado di modificare la forma degli E.

Una delle caratteristiche più peculiari degli $\mathrm{E}$ è la deformabilità che permette a queste cellule di passare anche attraverso capillari sanguigni il cui diametro è molto più piccolo del loro.

La deformabilità influisce direttamente sulla sopravvivenza degli E; la perdita di questa caratteristica rende estremamente più fragili gli $\mathrm{E}$ stessi $(1,2)$.

Nel paziente uremico, sottoposto ad emodialisi, gli $\mathrm{E}$ hanno una minore sopravvivenza, una minore deformabilità $(3,4)$ e sono perciò più suscettibili agli insulti meccanici.

La membrana plasmatica degli E, nell'insufficienza renale cronica, presenta delle modificazioni strutturali e funzionali che possono determinare, come già accennato, la comparsa di alterazioni morfologiche. Tra le alterazoni strutturali, forse la più importante è costituita da variazioni della componente lipidica (5), mentre, tra le alterazioni funzionali, riveste uno dei ruoli principali la diminuzione dell'attività della $\mathrm{Na}$, K-ATPasi che determina un incremento del $\mathrm{Na}$ negli $\mathrm{E}$ $(6,7)$.

Lo striscio periferico, in caso di uremia,

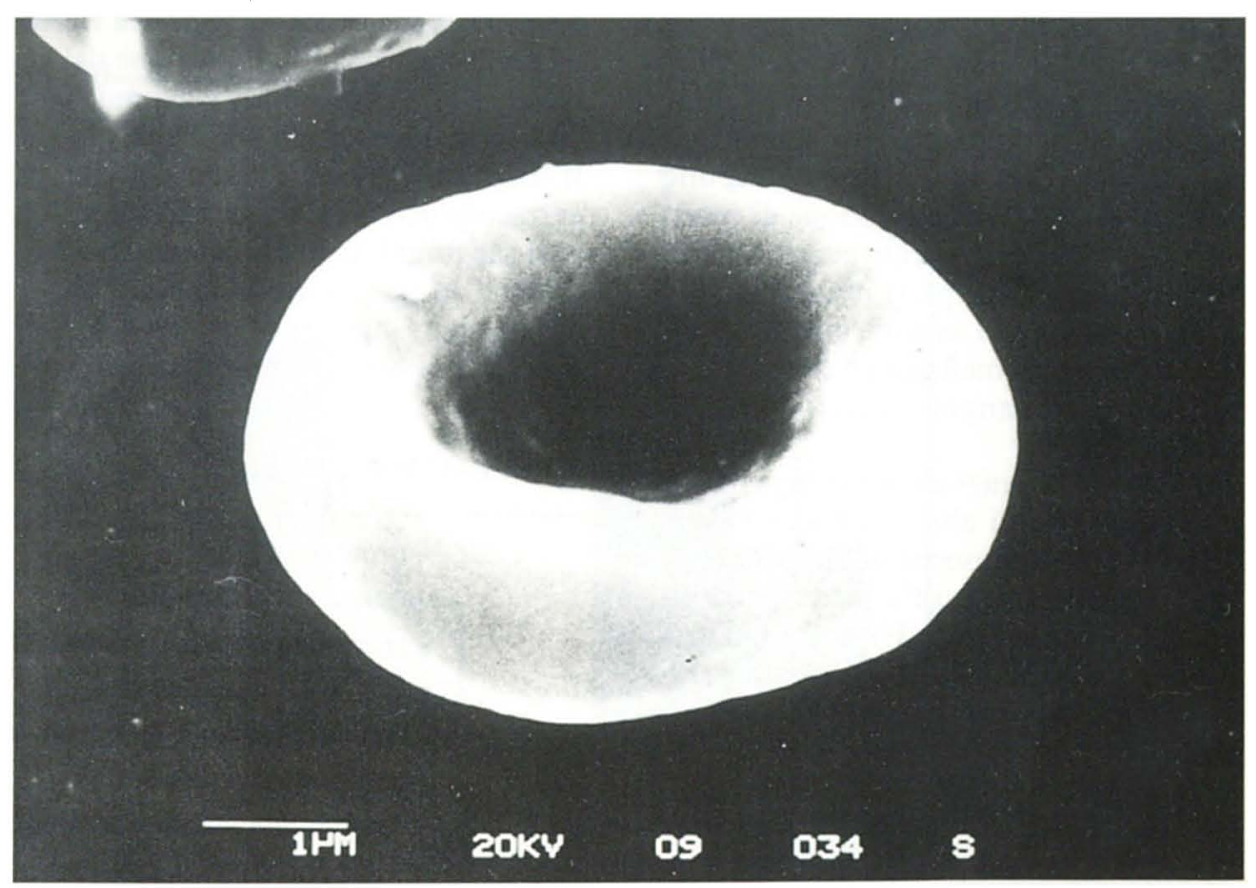

Fig. 1 - Eritrocita al microscopio elettronico a scansione. 


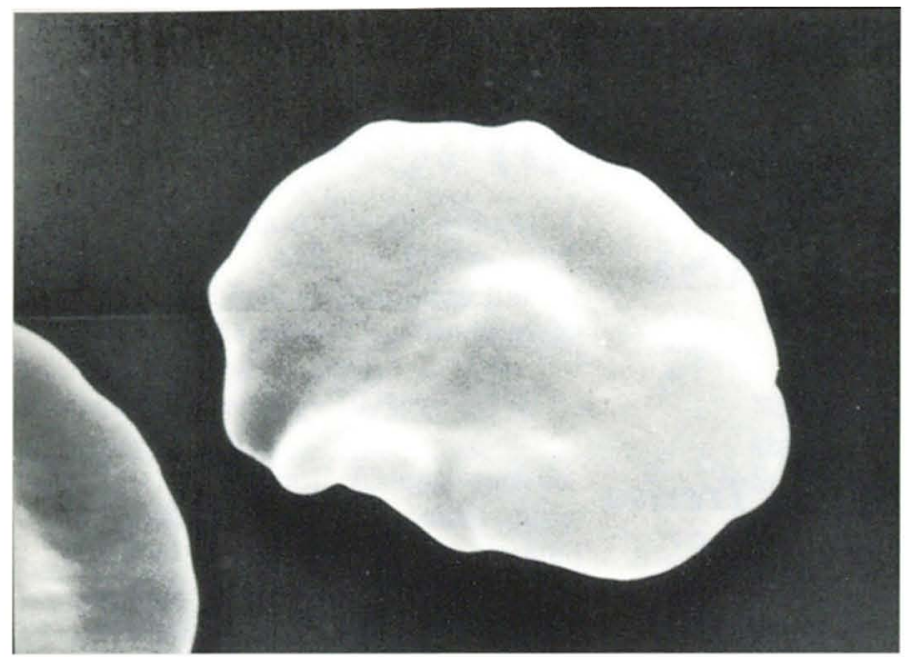

Fig. 2 - Echinocita di classe I al microscopio elettronico a scansione.

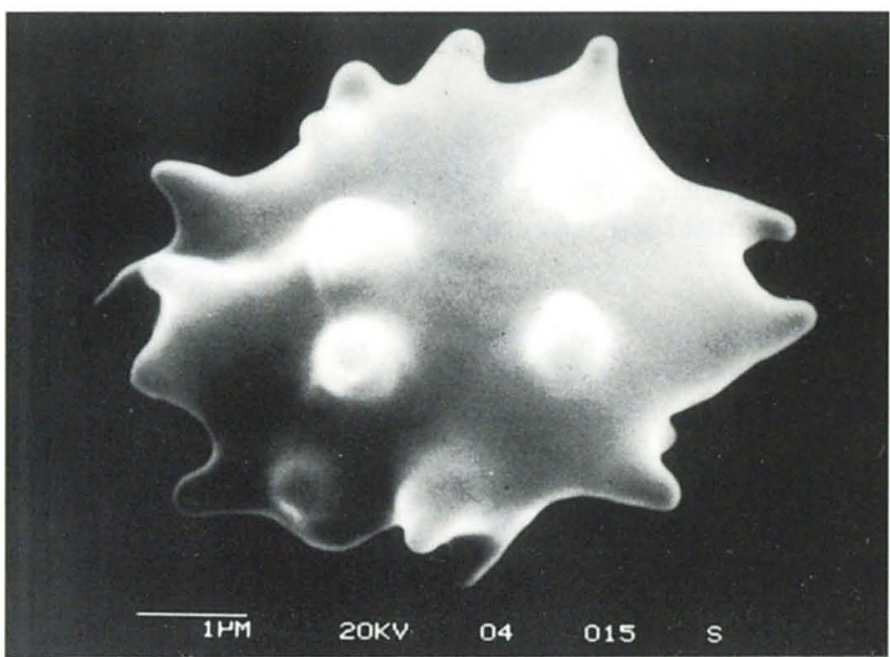

Fig. 3 - Echinocita di classe II al microscopio elettronico a scansione. evidenzia, generalmente, E normocitici e normocromici. Non si può, tuttavia, escludere la presenza di E microcitici ed ipocromici in caso di deficit di ferro (8) o quando si sviluppa una tossicità d'alluminio (9) o un quadro di macrocitosi nel caso, ad esempio, sia presente un deficit di folati (10). Spesso si osservano anche un certo numero di echinociti (E muniti di spicule) $(1,2)$ meglio evidenziabili al microscopio elettronico a scansione rispetto all'osservazione in microscopia ottica (Fig. 2-3).

Gli echinociti sono caratteristici, ma non specifici dello stato uremico. Infatti sono stati osservati, in vivo, anche in altre condizioni: nel deficit di piruvato-chinasi, come conseguenza di una deplezione in ATP; in talune anemie con diminuzione del potassio globulare; in alcune epatiti neonatali; dopo iniezione di eparina per effetto degli acidi grassi liberati dalla lipoprotein-lipasi; nel carcinoma dello stomaco; nel sanguinamento da ulcera peptica, immediatamente dopo trasfusione con sangue "vecchio" $(1,2)$ (Tab. I).

Altri tipi di alterazioni morfologiche sono espressione di altre patologie. Come, ad esempio, la presenza di sferociti (cellule che hanno perso l'aspetto tipico biconcavo dei normali E) nelle anemie emolitiche o di schizociti (frammenti cellulari irregolari) nelle anemie emolitiche micro-angiopatiche $(1,2)$.

In vitro, gli echinociti sono stati riscontrati in seguito ad una trasformazione dei discociti, nel sangue conservato o per artefatti, come quando vengono usati vetrini coprioggetto di vetro anziché di plastica (1).

La misurazione del volume degli E all'analizzatore ematologico H1 BayerTechnicon ha dimostrato la possibilità di evidenziare anche una certa quota di macrociti, imputabili ad una alterazione funzionale della membrana eritrocitaria, in particolare legata ad una riduzione dell'attività della pompa del sodio (11, 12). Dopo il trattamento emodialitico vi sarebbe una parziale correzione della macrocitosi, imputabile verosimilmente alla correzione dell'acidosi che influenza l'attività di trasporto sodico transmembrana $(11,13)$. Anche una nostra ricerca ha dimostrato una lieve riduzione del volume cellulare medio nei campioni postdialisi rispetto a quelli pre-dialisi (14). Il sangue scorre all'interno del circuito extracorporeo (ma anche all'interno dei vasi sanguigni) mantenendo un flusso laminare. In questo modo la parte corpuscolata del sangue (globuli rossi, globuli bianchi e piastrine) tende ad essere sepa- rata dal plasma e viene spinta verso la parete delle linee ematiche. Questa compartimentazione delle cellule ematiche comporta, per il ridursi della viscosità del sangue, una maggiore velocità al centro dell'area di scorrimento del sangue rispetto alla periferia. Il profilo della velocità del flusso ematico presenta infatti una configurazione parabolica.

Variazioni di calibro in qualsiasi punto del circuito extracorporeo, piegature delle linee, porte ematiche dei filtri (molto importanti nel determinare la distribuzione del flusso) non idonee e strutture dei filtri diverse possono essere cause in grado di trasformare il flusso laminare in un flusso vorticoso con possibilità di provocare una alterazione morfologica o un traumatismo più severo fino alla lisi degli elementi figurati del sangue. Tra questi le piastrine risultano le cellule più fragili seguite dai globuli e quindi dagli $\mathrm{E}$. Per quanto riguarda i filtri di dialisi, a parte vecchie segnalazioni legate al trauma meccanico subito dagli E nel passag-

TAB. I - CAUSE DI ECHINOCITOSI (IN VIVO)

$$
\begin{aligned}
& \text { - uremia } \\
& \text { - deficit piruvato-chinasi } \\
& \text { - riduzione potassio eritrocitario } \\
& \text { - talune epatiti neonatali } \\
& \text { - immediatamente dopo trasfusione di sangue "vecchio" } \\
& \text { - carcinoma gastrico } \\
& \text { - sanguinamento ulcera peptica } \\
& \text { - dopo iniezione d'eparina }
\end{aligned}
$$




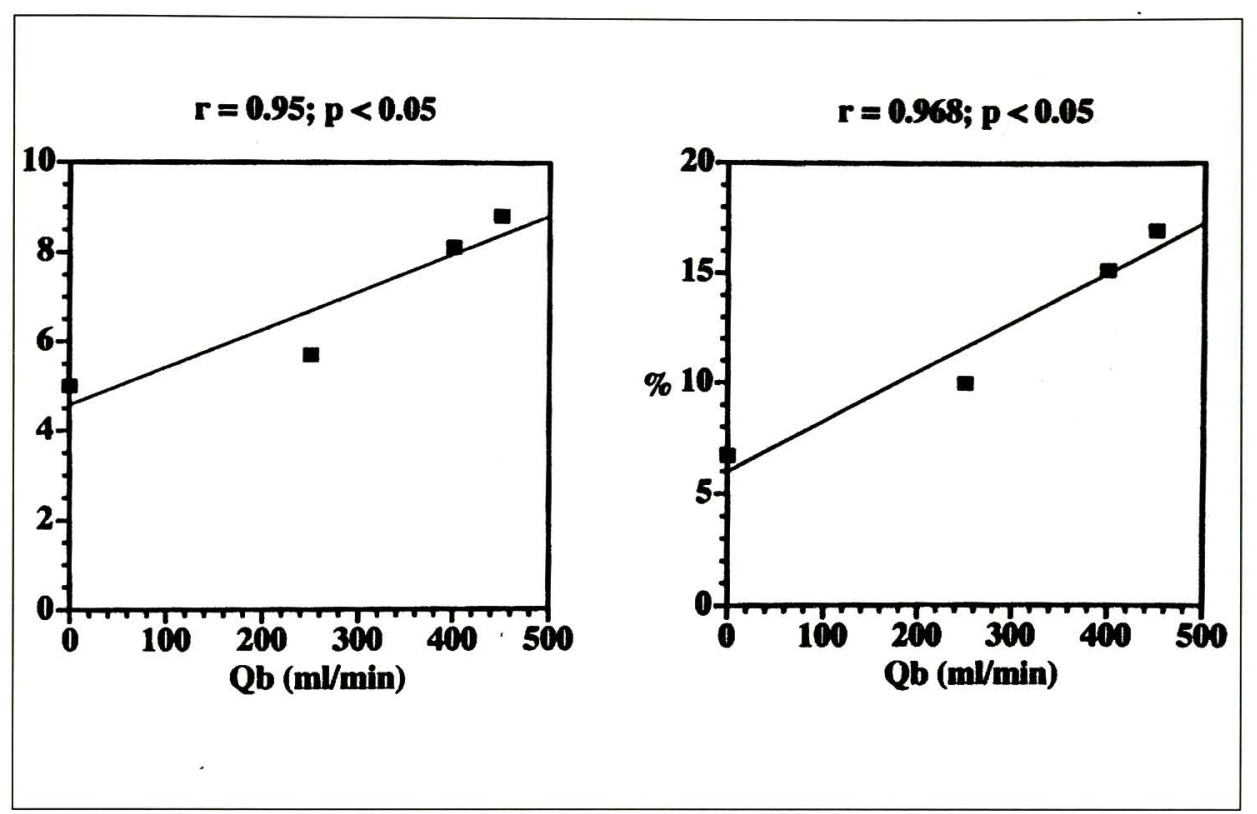

Fig. 4 - Correlazione tra aumento del flusso ematico $(Q b)$ e percentuale di emazie dismorfiche (a sin.: mediante l'applicazione della classificazione di Bessis; a dx.: con l'analizzatore d' immagini). gio attraverso dializzatori abbandonati da molti anni (15) ed alcuni episodi di emolisi attribuiti al riuso dei filtri (16), non sono note altre condizioni in grado di alterare, in modo significativo gli E.

Per quanto riguarda il tampone utilizzato nel trattamento emodialitico, invece, alcuni Autori (17) hanno documentato un maggior grado di danno eritrocitario nei pazienti trattati in acetato rispetto a quelli in bicarbonato.

La pompa peristaltica a rulli, mediante la sua azione di ritmica compressione sulle linee ematiche, può causare un danno cellulare sia per il cambiamento di flusso che determina, sia per il trauma meccanico diretto (ad esempio in seguito a malocclusione della pompa a rulli). A tale meccanismo finora non è stata data molta importanza, non avendo dimostrato gli studi condotti su questo argomento una particolare evidenza (18-22), salvo sottolineare il maggior rischio di emolisi nel sistema ago singolo doppia pompa soprattutto in caso di eccessive pressioni positive o negative e di altri flussi ematici che aumentano lo "shear stress" e le turbolenze (15, 23-25).

La tendenza ad incrementare i flussi ematici, mediante l'incremento degi giri della pompa, per ottenere dialisi sempre più efficienti, ci ha spinto a rivalutare il problema e ad eseguire alcuni studi allo scopo di evidenziare eventuali alterazioni di forma o traumatismi più gravi a ca- rico delle emazie.

In un recente lavoro (26) abbiamo documentato, al microscopio elettronico a scansione, in una prova in vitro, una correlazione diretta statisticamente significativa tra aumento del flusso ematico e percentuale di E alterati nella morfologia (echinociti), sia attraverso lo studio eseguito con 1'analizzatore d'immagini, sia mediante l'applicazione della classificazione di Bessis (Fig. 4).
In un altro studio (dati non ancora pubeventuali danni da catatere per emodialisi utilizzando una doppia pompa e confrontado varie situazioni: flussi ematici di $150 \mathrm{ml} / \mathrm{min}$, flussi ematici di 250 $\mathrm{ml} / \mathrm{min}$, flussi di $250 \mathrm{ml} / \mathrm{min}$ provocando volutamente una piegatura del catetere e flussi di $250 \mathrm{ml} / \mathrm{min}$ utilizzando una sola pompa e due aghi. Ebbene, abbiamo riscontrato un aumento statisticamente significativo delle emazie alterate nella morfologia (soprattutto echinociti) solo nella prova eseguita con il catetere piegato. D'altro canto altri Autori hanno già segnalato emolisi in caso di piegatura delle linee ematiche (27) o di parziale occlusione trombotica dei cateteri per emodialisi (28).

In un altra ricerca (29) abbiamo valutato gli eventuali effetti della pompa peristaltica addizionale presente nel circuito extracorporeo nel sistema della doppia filtrazione (30) sulla morfologia degli E osservati sempre in microscopia elettronica a scansione. I due trattamenti non differivano nella percentuale di emazie alterate nel pre e nel post-dialisi.

Abbiamo osservato una riduzione statisticamente significativa della percentuale degli E alterati nella forma (quasi esclusivamente echinociti) nel post-dialisi rispetto al pre sia nella dialisi eseguita con la doppia filtrazione, sia nella dialisi di controllo (Fig. 5). I risultati di questo studio parrebbero escludere, quindi, la blicati) abbiamo valutato in vitro gli

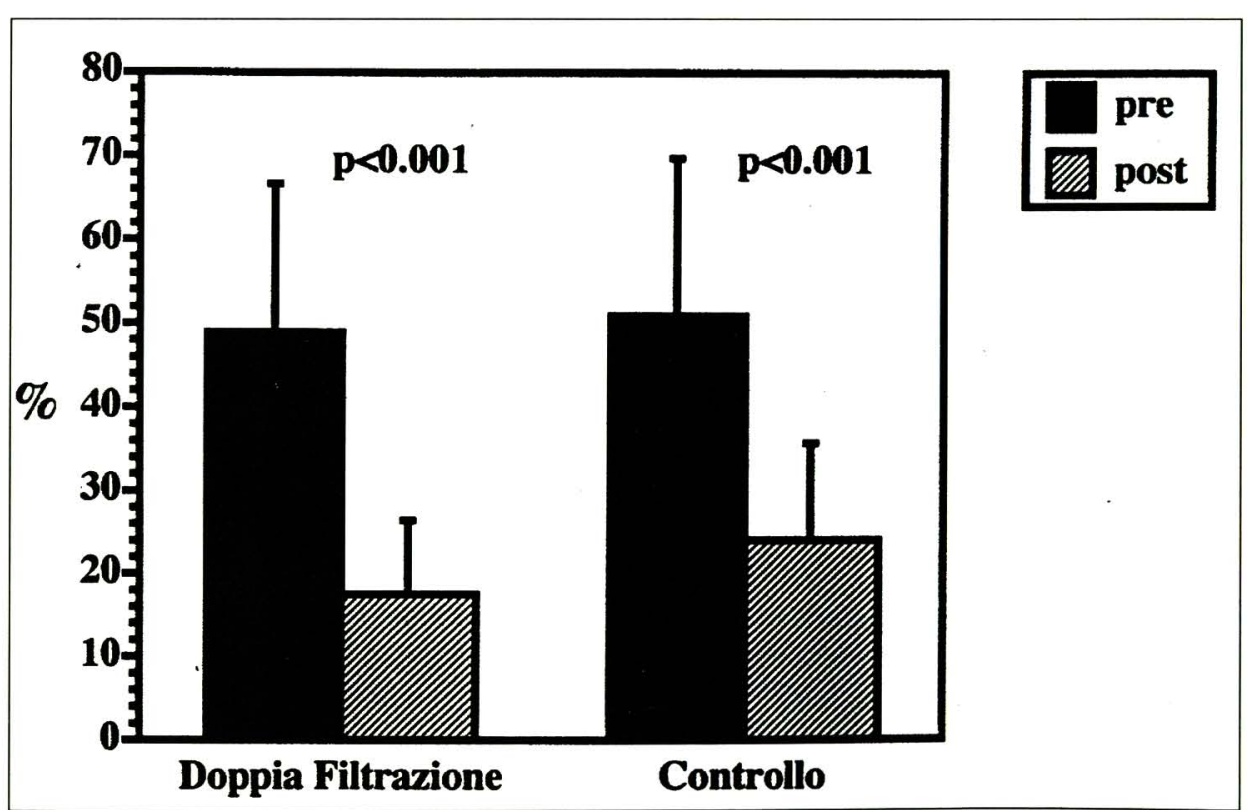

Fig. 5 - Percentuale di emazie dismorfiche, prima e dopo trattamento, con il sistema della doppia filtrazione e nella dialisi di controllo. 


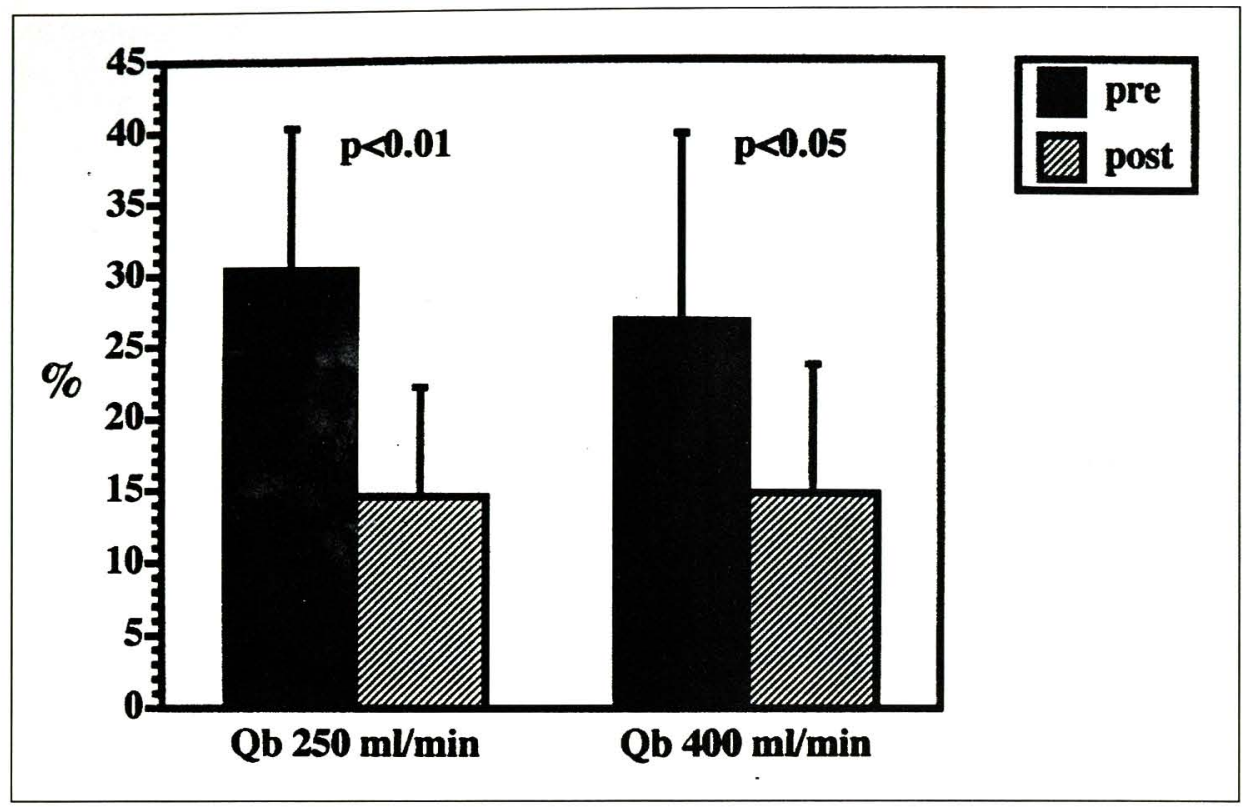

Fig. 6 - Percentuale di emazie dismorfiche, prima e dopo trattamento, a differenti flussi ematici.

possibilità che la pompa addizionale, presente nel sistema a doppia filtrazione, determini un danno più severo alle emazie rispetto alla dialisi standard con pompa singola.

La riduzione della echinocitosi nel postdialisi è in accordo con quanto da noi già pubblicato $(26,31)$ quando abbiamo confrontato, in una prova in vivo, negli stessi pazienti, dialisi a Qb di 250 $\mathrm{ml} / \mathrm{min}$ con dialisi a Qb di $400 \mathrm{ml} / \mathrm{min}$. Anche in questo confronto, infatti, riscontravamo un calo degli echinociti nel post-dialisi rispetto ai valori pre-dialisi a prescindere dal Qb applicato (Fig. 6).

Verosimilmente la dialisi, attraverso l'azione di rimozione di tossine uremiche e correggendo sia i disordini idroelettrolitici che l'acidosi crea le condizioni permittenti la trasformazione degli echinociti in discociti.

Insomma, da queste nostre ricerche, risulta come la dialisi, di per sè, non sia echinogena, ma anzi determini un calo degli echinociti che annullerebbe anche la potenziale azione lesiva data dalla pompa.

A sostegno di un miglioramento della crasi ematica nel post-dialisi vi sono in letteratura vari contributi. Il National Cooperative Dialysis Study ha dimostrato come i pazienti emodializzati con BUN più elevato presentassero un grado di anemia più severo di quelli con $\mathrm{BUN}$ più basso (32); alcuni Autori hanno di- mostrato, nei soggetti con insufficienza renale, una correlazione inversa statisticamente significativa tra ematocrito e creatininemia (33); altri Autori hanno evidenziato un miglioramento dell'anemia dopo l'inizio del trattamento emodialitico od il passaggio dalla dialisi bisettimanale a quella trisettimanale ed è stato suggerito come la dialisi sia in grado di allontanare degli "uraemic inhibitors" della eritropoiesi (34-36); infine è stato riportato come l'emodialisi ad alta efficienza determini un importante miglioramento dell'anemia (37). È lecito quindi ipotizzare come, a fine dialisi, la rimozione di BUN, della creatinina e di altri "uraemic factors" non meglio identificati, possa contribuire anche a ridurre il numero di echinociti. D'altro canto è stato riportato come la percentuale di echinociti nei pazienti con insufficienza renale sia in rapporto ai livelli di BUN e non alla gravità dell'anemia $(38$, 39).

La trasformazione discocito-echinocito (e viceversa) è reversibile.

Questa possibilità, peraltro già nota (1), è stata da noi dimostrata non solo tra il pre ed il post-dialisi, come già riportato nella prova in vivo (26), ma anche in altre situazioni come ad esempio, nella prova in vitro, dove confrontavamo vari flussi ematici, e dove abbiamo riscontrato la completa regressione delle alterazioni echinocitarie quando analizzavamo gli E dopo un intervallo di riposo di 15 minuti dalla fine della prova (26). È verosimile, che in quest'ultima condizione, lasciando il sangue a riposo, si creino le condizioni permittenti il ripristino della normale forma degli $\mathrm{E}$.

Per quanto riguarda il danno più grave che può subire un eritrocita, ovvero la sua frammentazione e la conseguente lisi cellulare, questa può verificarsi in varie situazioni, fortunatamente non frequenti: anomalie di membrana, deficit enzimatici, emoglobinopatie, emoglobinuria parossistica notturna, forme immuni, infezioni, grave ipofosfatemia, farmaci ( 25 , 27, 40). Episodi emolitici acuti sono stati descritti in corso di trattamento emodialitico causati da svariati fattori, oltre a quelli meccanici, già citati; contatto del sangue con dialisati contenenti zinco (41), rame (42), cloramine (con formazione di Heinz bodies) (43), nitrati (44), formaldeide (45), eccessivo riscaldamento (46) o ipo-osmolarità del bagno di dialisi (4)), residui di formaldeide per il riutilizzo dei filtri (16), residui di ipoclorito utilizzati per la sterilizzazione delle apparecchiature (48).

In conclusione la procedura emodialitica, salvo alcune situazioni sopra descritte, può essere assolta come causa di dismorfie eritrocitarie.

Una particolare attenzione, tuttavia, va ricercata quando si utilizza l'ago singolo o il catetere con la doppia pompa soprattutto in presenza di flussi ematici elevati. In questi casi è opportuno evitare l'impiego di aghi o cateteri di diametro interno piccolo e/o troppo lunghi che determinerebbero un incremento della resistenza al flusso ematico ed è utile provvedere ad un attento controllo delle pressioni nelle linee arteriose o venose. 


\section{BIBLIOGRAFIA}

1. Bessis M. Reinterpretazione degli strisci di sangue. Padova: Piccin Editore, 1982; 25.

2. Bull BS, Breton-Gorious J, Bentler E. Morphology of erythron. In: Williams WJ, Beutler E, Erslev AJ, Lichtman MA, eds. Hematology. 4th ed. London: McGraw-Hill Publishing Company, 1991; 297.

3. Kock KM, Patyna WD, Shaldon S, Werner E. Anemia of the regular hemodialysis patients and its treatment. Nephron 1974; 12: 40519.

4. Eschbach JW, Adamson JW. Anemia of end-stage renal disease (ESRD) Kidney Int 1985; 28: 1-5.

5. Cooper RA. Abnormalities of cell membrane fluidity in the pathogenesis of disease. N Engl J Med 1977; 297: 371-7.

6. Welt LG, Sachs JR, MacManus JJ. An ion transport defect in erythrocytes from uremic patients. Trans Assoc Am Phys 1964; 77: 169-81.

7. Stokes GS, Norris LA, Marwood JF, Johnston H, Caterson RJ. Effect of dialysis on circulating $\mathrm{Na}, \mathrm{KATPase}$ inhibitor in uremic patients. Nephron 1990; 54: 127-33.

8. Strickland ID, Chaput De Saintonge DM, Boulton FE, Francis B, Roubikova J, Waters JI. The therapeutic equivalence of oral and intravenous iron in renal dialysis patients. Clin Nephrol 1977; 7: 55-7.

9. Short AJK, Winney RJ, Robson JS. Reversible microcytic hypochromic anemia in dialysis patients due to aluminum intoxication. Proc Eur Dial Transplant Assoc 1980; 17: 233-6.

10. Hampers CL, Streiff R, Nathan DG, Snyder D, Merrill JP. Megaloblastic hematopoiesis in uremia and in patients on long-term he- modialysis. N Engl J Med 1967; 276: 551-4.

11. Enzmann G, Gianoli E, Ianche M, Piccinini C, Panzetta G. Anemia macrocitica normocromica nel paziente in dialisi. Giorn It Nefrol 1994; 11: 63-5.

12. Pascucci M, Canestrari P, Galli $\mathrm{F}$, Albertini MC, Bigiarelli B, Galiotta P. Stress eritrocitario nel trattamento dialitico extra-corporeo: effetti sul trasporto di membrana. Giorn It Nefrol 1993; 10: 81-90.

13. Levin ML, Rector FC, Seldin DW. The effect of chronic hypokalemia, hyponatremia and acid-base alterations on erythrocyte sodium transport. Clin Sci 1972; 43: 251-63.

14. Da Rin G, De Angeli $S$, Calzavara P, Caberlotto L, Tessarolo A, Savelli L, Caenaro GF. Variazioni morfologiche e parametriche dei globuli rossi all'H* 1 . Atti $5^{\circ}$ incontro club utilizzatori sistemi ematologici Bayer-Technicon 1991; 25-30.

15. Blackshear PL Jr, Dorman FD, Steinbach JH. Some mechanical effects that influence hemolysis. Trans Am Soc Artif Intern Organs 1965; 11: 112-7.

16. Cheung AK. Membrane biocompatibility. In: Nissenson AR, Fine RN, Gentile DE. Clinical dialysis 2nd ed. East Norwalk, Connecticut: International Edition 1990: 69-96.

17. Hampl H, Riedel E, Wendel G, Scigalla P. Red blood cell density distribution in uremic patients on acetate and bicarbonate hemodialysis. Blood Purif 1990; 8: 260-7.

18. Keshavian P, Leuhmann D, Shapiro F, Comty C. Investigation of the risks and hazards associated with hemodialysis systems. US Dept of Health and Human Services. Silver Spring, Public Health Service/FDA/Bureau of Medical Devices, 1980: 184.

19. Bernstein EF, Indeglia RA, Shea MA, Varco RL. Sublethal damage to the red blood cell from pumping. Circulation (Suppl) 1967; 1: 226-33.

20. Hyde SE, Sadler JH. Red blood cell destruction in hemodialysis. Trans Am Soc Artif Intern Organs 1969; 15: 50-4.

21. Keshaviah PR, Shaldon S. Haemodialysis monitors and monitoring. In: Drukker W, Parsons FM, Maher JF. Replacement of renal function by dialysis 2 nd ed. The Hague: Martinus Nijhoff, 1983; 223.

22. Lerner R, Werner B, Asaba H, Ternstedt B, Elmquist E. Assessment of hemolysis in regular hemodialysis patients by measuring carbon monoxide production rate. Clin Nephrol 1983; 20: 239-43.

23. Dhaene M, Gulbis B, Lietaer N, Gammar N, Thayse C, Ooms HA, Vanherweghem JL. Red blood cell destruction in single-needle dialysis. Clin Nephrol 1989; 31: 327-31.

24. Bernstein EF, Blackshear PL, Keller KH. Factors influencing erythrocyte destruction in artificial organs. Am J Surgery 1967; 114 : 126-38.

25. Schuett H, Port FK. Hemolysis in hemodialysis patients. Dial Transplant 1980; 9: 345-7.

26. Calzavara P, De Angeli S, Nieri A, Furlan C, Bolzonella R, da Porto A. Alterations in erythrocyte morphology induced by blood pumps. Int J Artif Organs 1993; 16: 6538 .

27. Gault MH, Duffett S, Purchase L, Murphy J. Hemodialysis intravascular hemolysis and kinked blood lines. Nephron 1992; 62: 267-71.

28. Nand S, Bausal VK, Kozeny G, Vertuno L, Remlinger KA, Jordan JV Jr. Red cell fragmentation syndrome with the use of subclavian hemodialysis catheters. Arch Intern Med 1985; 145: 1421-3.

29. Calzavara P, De Angeli S, Furlan C, da Porto A, Calconi G. Double filtration does not damage erythrocytes. Int J Artif Organs 
(in stampa).

30.

Brendolan A, Ronco C, Crepaldi C, Bragantini L, Milan M, Gastaldon F, La Greca G. Double pass dialysis: a new method of renal replacement in patients with malfunctioning vascular access. Int $\mathbf{J}$ Artif Organs 1994; 17: 379-84.

31. Calzavara P, Amici G, Calconi G, De Angeli S, Da Rin G, Furlan C. Reduction of echinocytes posthemodialysis. Nephrol Dial Transplant (in stampa).

32. Santiago GC, Sreepada Rao TK, Laird NM. Effect of dialysis therapy on the hematopoietic system: The NCDS. Kidney Int (Suppl 13) 1983 ; 23: S95.

33. McGonigle RJS, Wallin JD, Shadduck RK, Fisher JW. Erythropoietin deficiency and inhibition of erythropoiesis in renal insufficiency. Kidney Int 1984; 25 : 437-44.

34. Radtke HW, Frei U, Erbes PM, Shoeppe W, Koch KM. Improving anemia by hemodialysis: effect on serum erythropoietin. Kidney Int: 1980; 17: 382-7.

35. Eschbach JW, Adamson JW, Cook JD. Disorders of red blood cells production in uremia. Arch Intern Med 1970; 126: 812-5.

36. Radtke HW, Claussner A, Erbes PM, Scheuermann EH, Schoeppe W, Koch KM. Serum erythropoietin concentration in chronic renal failure: relationship to degree of anemia and excretory renal function. Blood 1979; 54: 87784.

37. Mastrangelo F, Alfonso L, Rizzelli S, Aprile M, Sandri L. Anaemia in high-efficiency dialysis. Nephrol Dial Transplant 1991; Suppl 2: 116-21.

38. Cooper RA. The pathogenesis of burr cells in uremia. J Clin Invest 1970; 49: 22A.

39. Cooper RA, Shattil SJ. Mechanisms of hemolysis. The minimal red cell defect. N Engl $\mathbf{J}$ Med
1971; 285: 1514-20.

40. Jacob HS, Amsden T. Acute hemolytic anemia with rigid red cel1s in hypophosphatemia. $\mathrm{N}$ Engl J Med 1975; 285: 1446-50.

41. Petrie JJ, Row PG. Dialysis anaemia caused by subacute zinc toxicity. Lancet 1977; 1: 1178-80.

42. Manzler AD, Schreiner AW. Copper induced acute hemolytic anemia. A new complication of hemodialysis. Ann Intern Med 1970; 73: 409-12.

43. Eaton JW, Kolpin CF, Swofford HS, Kjellstrand CM, Jacob HS, Chlorinated urban water: A cause of dialysis-induced hemolytic anemia. Science 1973; 181: 463-4.

44. Salvadori M, Martinelli F, Comparini L, Bandini S, Sodi A. Nitrate induced anaemia in home dialysis patients. Proc Eur Dial Transplant Assoc 1984; 21: 321-5.

45. Orringer EP, Mattern WD. Formaldehyde induced hemolysis during chronic hemodialysis. N Engl J Med 1976; 294: 1416-20.

46. Berkes SL, Kahn SI, Chazan JA, Garella S. Prolonged hemolysis from ovrheated dialysate. Ann Intern Med 1975; 83: 363-4.

47. Said R, Quintanilla A, Levin $\mathrm{N}$, Ivanovich P. Acute hemolysis due to profound hypo-osmolality. A complication of hemodialysis. J Dial 1977; 1: 447-52.

48. Hoy RH. Accidental systemic exposure to sodium hypochlorite (Clorox) during hemodialysis. Am J Hosp Pharm 1981; 138: 1512-4. 